\title{
Fuzzy logic-driven expert system for the diagnosis of heart failure disease
}

\author{
Akinyokun O. C. ${ }^{* 1}$, Iwasokun G. B. ${ }^{2}$, Arekete S. A. ${ }^{3}$, Samuel R. W. ${ }^{2}$ \\ ${ }^{1}$ Dept. of Physical Sciences, Landmark University, Omuaran, Nigeria \\ ${ }^{2}$ Federal University of Technology, Akure, Nigeria \\ ${ }^{3}$ Redeemer's University, Redemption City, Sagamu, Nigeria
}

Received: September 9, 2014

DOI: $10.5430 /$ air.v4n1p12
Accepted: October 30, $2014 \quad$ Online Published: January 1, 2015

URL: http://dx.doi.org/10.5430/air.v4n1p12

\begin{abstract}
The heart is a key organ of the human system which pumps and circulates blood throughout the body. Its failure to perform these functions often leads to total breakdown of the entire body system and in most cases results in death. In several countries of the world, significant numbers of heart failure patients are being reported dead due to inaccurate and untimely diagnosis. Recently, expert system approach, which is anchored on Information Technology, has emerged as a strong tool for solving this problem. This paper reports on the development of a Web and Fuzzy Logic-based Expert System for the diagnosis of heart failure disease. The proposed system consists of a Knowledge Base (which is made up of a Database), a Fuzzy Logic component, a Fuzzy Inference Engine and a Decision Support Engine which comprises of cognitive and emotional filter as well as Tele-medicine facilities. The system was implemented using Hypertext Preprocessor (PHP), JavaScript and Hypertext Mark-up Language (HTML) with My Structured Query Language (MySQL) as the Database Management System. Performance analysis based on data on selected heart failure patients and survey of some experts on heart failure disease at the State Specialist Hospital, Akure, Nigeria shows satisfactory performances of the system.
\end{abstract}

Key Words: Heart failure, Expert system, Fuzzy logic, Diagnosis, Medical expert

\section{Introduction}

The limitations of the conventional methods for the diagnosis of diseases call for the development of expert systems which will aid medical practitioners in delivering effective and efficient medical services to patients at affordable prices (cost) irrespective of their geographical location. Due to the strength of Fuzzy Logic (FL) in the provision of accurate solutions to difficult real life problems and the advancement in Internet technology, there has been an increasing need to incorporate FL concept into medical diagnosis for a successful development of Internet-based expert system that will have a human-like reasoning capability.
Cardiovascular disease is the class of diseases that involves the heart or blood vessels (arteries and veins). While the term technically refers to any disease that affects the cardiovascular system, it is generally used to refer to those related to atherosclerosis (arterial disease). Cardiovascular system, otherwise known as circulatory system in human, carry out the combined function of the heart, blood and blood vessels to transport oxygen and nutrients to organs and tissues throughout the body and carry away waste products, amongst its vital functions. Diseases of the cardiovascular system include those that compromise the pumping ability of the heart, cause failure of the valves or result in narrow-

\footnotetext{
*Correspondence: Akinyokun O. C.; Email: akinwole2003@yahoo.co.uk; Address: Dept. of Physical Sciences, Landmark University, Omuaran, Nigeria
} 
ing/hardening of the arteries. The normal function of the heart is the pumping of blood and circulating it throughout all parts of the body. When the heart's output cannot meet the normal work, a condition known as heart failure occurs and when the heart fails, other vital organs such as the Kidney, Lungs, and Liver are also affected. Heart failure disease and the resulting complications are the main cause of death for both males and females in most technologically advanced countries of the world. It is also rated among the top five causes of death in lesser-developed countries. ${ }^{[1,2]}$

Medical diagnosis involves identifying illness or disorder in a patient through physical examination, medical tests or other procedures while therapy is the treatment of physical, mental or behavioral problems and it is meant to cure or rehabilitate the sick. ${ }^{[3-11]}$ The management of heart failure disease is highly essential and involves considerable amount of medical records which have to be collected, cleaned, stored, selectively retrieved and statistically analyzed. This could not be efficiently handled by the traditional method that is in practice among health practitioner. Other militating factors include inadequate number of healthcare delivery experts, high cost in terms of finance associated with the delivery of quality healthcare services by medical experts and limited memory capability, low retention of information and untimely recall of accurate information which is very common among medical practitioners due to the nature of their job. Others are insecure patient medical records and wrong decisions seldom made by medical expert due to fatigue, emotional conditions and other human factors.

The diagnosis of heart failure falls into three basic categories, history taking, physical examination and laboratory investigation. ${ }^{[1]}$ History taking involves obtaining a complete clinical (medical) history of the patient so as to deduce the symptoms. Physical examination by a cardiologist involves accessing the patient's general appearance for evidence of resting dyspnea, cyanosis and cachexia. It involves examining the blood pressure and heart beat, pulmonary, third and fourth heart sound and jugular venous distention. Laboratory findings involve reports on the patient's chest Xray, heart tracing Electrocardiogram (ECG), heart imaging (echocardiogram) and the likes.

The research being reported developed a web-based expert system for eliminating the limitations of some of the existing systems for the diagnosis, therapy and management of heart failure. The functionalities of the system was accessed by using data on heart failure of selected patients at a Specialist Hospital in Akure, Nigeria.

\section{Related works}

The authors in Ref.12 proposed a knowledge based system for the diagnosis and administration of tropical diseases. The system has functionalities for addressing problems of geographical mal-distribution of medical practition- ers and material resources, inadequate facilities to support the privacy and security of patients' medical records and prolonged delay of reports generation and statistical analysis of medical records. However, the system lacks the capability for global access due to its offline nature and could not handle vague (imprecise) data which are inherent in medical records. A decision support system for the diagnosis of tropical diseases using FL was proposed in Ref.13. The system provides medical personnel with a means for better diagnosis of tropical diseases and handles the problem of imprecise data mostly found in medical records. It however lacks enhancing features such as chat and e-mail facilities which are essential for instant communication among specialists from different part of the world during cases of difficult patient diagnosis.

The authors in Ref.14 presented a multiplayer perceptionbased decision support system for diagnosis of heart diseases. The input layer of the system includes variables categorized into 3 four groups and the number of nodes in the hidden layer is determined through a cascade learning process. Each of the nodes in the output layer corresponds to one heart disease of interest. The missing data of a patient were handled using the substituting mean method and an improved back propagation algorithm was used to train the system. Medical records collected from the sampled patients suffering from five heart diseases were used to train and test the system while the generalization assessment was by cross validation, holdout and bootstrapping. The authors in Ref.15 used many different types of data, including family and patient histories, laboratory results, physical findings, genetic information, electrocardiogram analysis and imaging results to formulate a computer-assisted decision support system with a variety of reasoning methodologies for the diagnosis of cardiac disorders. The authors in Ref.6 used the Case Based Reasoning (CBR) methodology to develop an expert system prototype for supporting diagnosis of heart diseases. Sampled data on two hundred and seven attributes from demographic and clinical data were collected for four heart diseases namely; mitral stenosis, left-sided heart failure, stable angina pectoris and essential hypertension. The analysis of these data was based on induction and nearest-neighbour retrieval strategies with the nearest neighbour outperforming the induction strategy.

A Fuzzy Expert System for heart disease diagnosis based on the V.A. Medical Center, Long Beach and Cleveland Clinic Foundation database is presented in Ref.16. The system has 13 input fields and one output field. Input fields are chest pain type, blood pressure, cholesterol, resting blood sugar, maximum heart rate, resting electrocardiography (ECG), exercise, old peak (ST depression induced by exercise relative to rest), thallium scan, sex and age. The output field is an integer valued representing the heart disease status which ranges from 0 (no presence) to 4 (distinguish presence). A neuro-fuzzy expert systems for the diagnosis and therapy of 
cardiovascular diseases were proposed. Fuzzy rules were formulated and used to implement the fuzzy logic diagnosis of heart failure with the Root Sum Square (RSS) inference procedure on a four layered feed forward Neural Networks based on the Sigmoid Activation functionA. ${ }^{[17,18]}$

The authors in Ref.9 presented a knowledge-based intelligent system for the diagnosis of "red eye" based on common Knowledge Acquisition and Documentation Structuring (KADS) methodology. The system has stages for identifying, specifying and refining knowledge. Its platform for designing the domain ontology and model involves revision and reusing knowledge from different sources. The authors in Ref.10 presented an intelligent hybrid system for diagnosing common diseases with four different architectures namely standalone, transformational, hierarchical and integrated. The standalone model consists of independent software components, which do not interact in any way and provides direct means of computing the problem solving capabilities of different techniques with references to certain applications. The transformational model begins as one type of system (such as a neural network) and end up as an expert system. The different layers in hierarchical architecture is associated with different functions while an integrated hybrid model combines different techniques into one single computational model which shares data structures and knowledge representations.

In Ref.21, a personal health assistance service expert system for advice on healthy food and activities for good practices of fitness and stable health was proposed. The system also provide basis for tracking personal health data and medical treatments and a comprehensive exchange of data. A medical diagnosis system that is based on fuzzy-neural network is presented in Ref.22. The main focus of the system is to ease the job of medical practitioners and as well provide accurate diagnosis result. Multilayer Perception (MLP) neural network and 4 ordinary back propagation GDR training algorithms were used. Though $97.5 \%$ accurate in diagnosis was reported, it failed to consider the calculation of the best membership function for each fuzzy data which significantly reduces its efficiency. The authors in Ref.23 proposed a Fuzzy Expert System for the Management of Malaria which has been identified as a predominant environmental health problem in several parts of the world. The system is suitable to act as a decision support platform to researchers, physicians and other healthcare practitioners in malaria endemic.

\section{Proposed neuro-fuzzy-based expert sys- tem for diagnosis}

The main components of the proposed Fuzzy Expert System are knowledge base, decision support engine and the World Wide Web as shown in Figure 1.

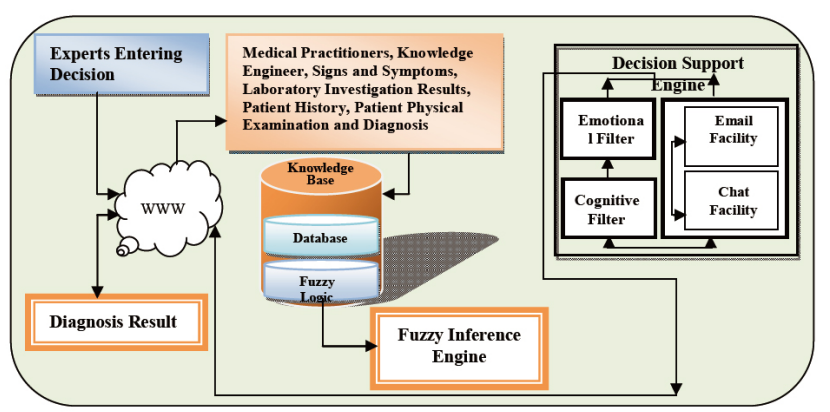

Figure 1: Components of the proposed system

\subsection{Knowledge base}

The Knowledge base stores static and dynamic knowledge of the diagnosis of heart failure. It serves as the information store for operational data to be processed and as well consists of database of quantitative knowledge and fuzzy logic of the qualitative knowledge of medical practitioner in heart failure diagnosis. The database of quantitative knowledge is a repository where patients' attributes presented in Table 1 needed for successful diagnosis are stored or retrieved.

The database model considered for the diagnosis of heart failure disease has its schema presented as follows based on the codes presented in Table 1.

Table 1: Heart Failure Diagnosis Attributes

\begin{tabular}{llll}
\hline S/N & Category & $\begin{array}{l}\text { Heart Failure Diagnosis } \\
\text { Attributes }\end{array}$ & Code \\
\hline 1 & & Rest Dyspnea & RD \\
2 & & Orthopnea & OP \\
3 & Patient Medical & Nocturnal Dypsnea & ND \\
4 & History & Dypsnea on Exertion & DE \\
5 & & Dypsnea While Climbing & DC \\
6 & & Heart Rate Abnormality & HR \\
7 & & Juvular Venous Elevation & VE \\
8 & Patient Physical & Lung Crackles & LC \\
9 & Examination & Wheezing & WZ \\
10 & & Third Heart Sound & HS \\
11 & & Alveolar Pulmonary Edema & AP \\
12 & Patient & Interstitial Pulmonary Edema & IP \\
13 & Laboratory & Bilateral Plural Effusion & BP \\
14 & Investigation & Cardiothoracic Ratio & CR \\
15 & & Upper Zone Flow & ZF \\
\hline
\end{tabular}

(1) Patient-Bio-Data (Patient-ID, Patient-Name, PatientAddress, Patient-Age, Patient- Sex, Nationality, Marital-Status, Blood-Group, Name-of-Next-of-Kin, Address-of- Next-of-Kin).

(2) Patient-Medical-History (Symptom-ID, Patient-ID, RD, OP, ND, DE, DC).

(3) Patient-Physical-Examination (Sign-ID, Patient-ID, HR, VE, LC, WZ, HS).

(4) Lab-Investigation (Lab-Test-ID, Patient-ID, AP, IP, BP, CR, ZF, Date-of-Exam). 
(5) Patient-Consultation (Patient-ID, Date-of-Diagnosis, Diagnosis).

\subsection{Fuzzy logic of heart failure diagnosis}

Fuzzy logic is a superset of the conventional Boolean logic with capability for handling imprecise (vague) and incomplete data that are commonly found in medical records. It resembles human decision making with its ability to work from approximate reasoning and ultimately find a precise solution to a given problem. The process of diagnosing heart failure by the fuzzy logic involves the following stages:

(1) Fuzzification of input variables (values of signs, symptoms, and laboratory test results).

(2) Establishment of the fuzzy rule base.

(3) Building the decision making logic of the fuzzy logic component (inference engine).

(4) Defuzzification of the output of the inference engine into crisp values.

Figure 2 presents the basic architecture of the fuzzy logic system for the diagnosis of heart failure and its flow of data/information.

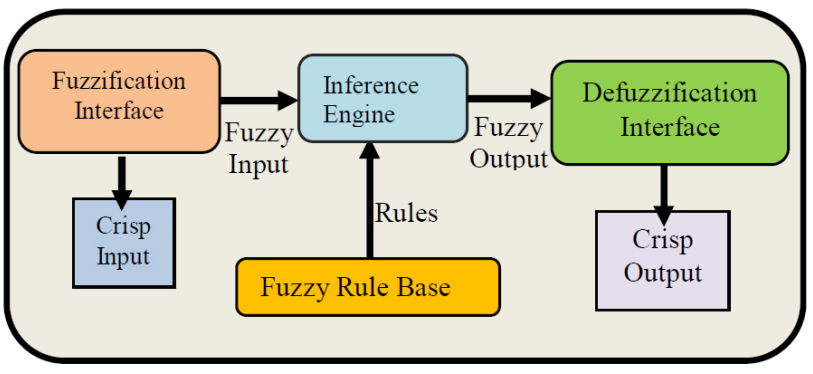

Figure 2: Basic Architecture of a Fuzzy Expert System

A fuzzy set healthy, mild, moderate, severe, very severe for the input variables (signs, symptoms, and laboratory test results) is defined. The input variables are fuzzified and the membership functions defined for them are applied to their actual values to determine the degree of truth for each rule antecedent. Given set $\mathrm{A}=\left[x_{1}, x_{2}, x_{3}, \cdots, x_{n}\right]$ which denotes input variables (signs, symptoms, test results), A is a collection of ordered pairs as follows:

$$
A=\left(x_{i}, \mu_{A}\left(x_{i}\right)\right), i=1,2,3, \cdots, n
$$

$\mu_{A}\left(x_{i}\right)$ is a triangular shaped membership function (MF) represented by real number in the interval $[0,1]$. The MF is defined as follows:

$$
\mu_{A}\left(x_{i}\right)=\left\{\begin{array}{cc}
0 & \text { if } x_{i} \leq a \\
\frac{x_{i}-a}{b-a} & \text { if } a \leq x_{i}<b \\
\frac{c-x_{i}}{c-b} & \text { if } \leq \leq x_{i}<c \\
0 & \text { ifc } \leq x_{i}
\end{array}\right.
$$

Published by Sciedu Press $a, b$, and $c$, are parameters of the membership function governing MF triangular shape. Each of the attributes (variables) is described by a linguistic term in the fuzzy set healthy, mild, moderate, severe, very severe.The input and output parameters were defined based on linguistic variables and their associated fuzzy value range are presented in Table 2. The corresponding MF graph is represented in Figure 3.

Table 2: Linguistic Variables and their Fuzzy Value Range

\begin{tabular}{llll}
\hline S/N & Linguistic Variables & Fuzzy Value & Input value \\
\hline 1 & Healthy & $0.0 \leq x<0.2$ & 4 \\
2 & Mild & $0.2 \leq x<0.4$ & 3 \\
3 & Moderate & $0.4 \leq x<0.6$ & 2 \\
4 & Sever & $0.6 \leq x<0.8$ & 1 \\
5 & Very Severe & $0.8 \leq x \leq 1.0$ & 0 \\
\hline
\end{tabular}

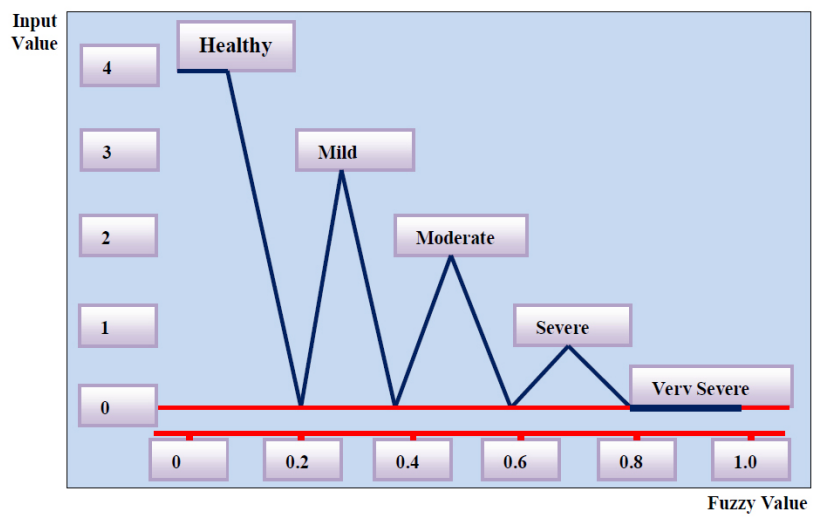

Figure 3: Membership Function Graph

\subsection{Fuzzy rule base for heart failure diagnosis}

The fuzzy rule base is characterized by a set of "If-then" rules, in which the antecedents ('If' part of the rule) and consequents ('then' part of the rule) involve linguistic variables. The collection of these rules forms the rule base for the fuzzy logic sub-system. The rules are carefully formulated based on the view of medical experts on diagnosis of heart failure disease. A structure of rules in the rule base is: if $(x=A)$ and $(y=B)$ and $(z=C)$ then $(S=O)$, where $x, y, z$ are inputs variables, $A, B, C$ are fuzzy sets of the input variables, $S$ is the output and $O$ is fuzzy set of the output variable within the fuzzy region specified by the rule.

\subsection{Inference engine of heart failure diagnosis}

The Inference Engine presents the decision making logic of the fuzzy logic sub-system. It receives input from the fuzzy rule base and the fuzzification interface components. It applies suitable composition procedure to its inputs in order to produce the desired output. The Inference Engine driven by Root Sum Square (RSS) inferential mechanism is of the 
form: $R_{K}$ :if [( $D C$ is mild) and ( $H R$ is severe)] then [(heart failure is moderate)].

"DC" and "HR" are inputs variables while "mild" and "severe' are fuzzy sets with input values 0.8 and 0.4 respectively. "heart failure" is the output and it is based on the conjunction of the two fuzzy sets while "moderate" is the fuzzy set (with input value of 0.6) of the output variable within the fuzzy region specified by the rule and it is obtained by rounding off the mean of the input values for the fuzzy sets to its nearest input value. RSS method is used to draw inferences by combining the effects of all fired rules and their respective magnitudes and compute the mean (composite area). This approach gives the best weighted influence to all firing rules.

If there are $R_{K}$ rules in the rule base, where $k=$ $1,2, \cdots, n$. The output signals of the rules are represented by the set: $\left\{O\left(R_{1}\right), O\left(R_{2}\right), O\left(R_{3}\right), \cdots, O\left(R_{n}\right)\right\}$. where $O\left(R_{k}\right) \in[0,1]$. Finally, the fired rule which represents the outcome of a particular diagnostic task are combined by using the formula:

$$
R S S=\sum_{k=1}^{n}\left(R_{k}^{2}\right)
$$

Due to the fact that the output from the inference engine is usually a fuzzy set, the output is translated into crisp values based on the fuzzy set. The translation of the inference engine is based on the Centroid of Area (CoA) technique as follows:

$$
C_{O} A=\frac{\left(\sum_{1}^{n} \mu_{Y}\left(x_{i}\right) x_{i}\right)}{\left(\sum_{1}^{n} \mu_{Y}\left(x_{i}\right)\right)}
$$

where $\sum_{1}^{n} \mu_{Y}\left(x_{i}\right)$ and $x_{i}$ are the membership value and center of MF respectively. Table 3 presents the linguistic values and the range of crisp values.

Table 3: Linguistic Variables and range of crisp value

\begin{tabular}{lll}
\hline $\mathbf{N}$ & Linguistic Variables & Crisp Value \\
\hline 1 & Healthy & $0.0 \leq \mathrm{x}<20$ \\
2 & Mild & $20 \leq \mathrm{x}<40$ \\
3 & Morderate & $40 \leq \mathrm{x}<60$ \\
4 & Severe & $60 \leq \mathrm{x}<80$ \\
5 & Very Severe & $\geq 80$ \\
\hline
\end{tabular}

\subsection{Decision support engine for heart failure diag- nosis}

The Decision support engine (DSE) consists of cognitive and emotional filters as well as tele-medicine engine. The output of fuzzy inference engine serves as input to the cognitive filter, while the output of the cognitive filter serves as input to the emotional filter. The cognitive filter employs the objective feelings of the medical practitioner to reduce the alternative output report of the inference engine. Typi- cal objective feelings include the decision to conclude that a patient has heart failure after diagnosis because the patient takes alcohol and smoke cigarette even if he has limited symptoms of heart failure. The emotional filter takes charge of the subjective feelings of the medical practitioner to reduce the alternative output report of the cognitive filter. Typical subjective feelings of a medical expert when diagnosing a patient is the decision to place a patient on bed rest over a period of time to further observe the symptoms of the disease, probably because the patient has not been having sufficient rest due to the nature of his/her job.

The tele-medicine component of the architecture consists of chat module and electronic mailing modules. The electronic mailing module promotes communication on diagnosis challenges via email among experts while the live chat module enables a medical expert to initiate a real life communication with an expert colleague in any other part of the world to seek his or her assistance regarding any diagnosis challenges. The platform for the live chat module is conceptualized in Figure 4. The Tele-medicine facilities enhance the overall performance of human experts and foster the production of valid, accurate, and timely diagnosis in a collaborative diagnosis and therapy of heart failure.

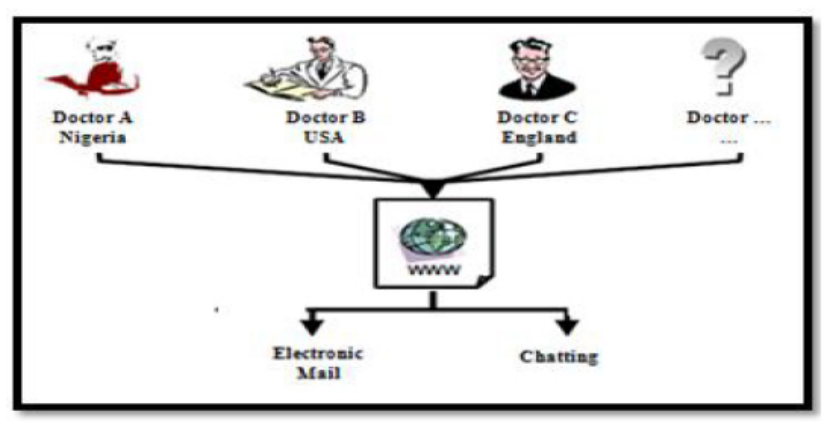

Figure 4: Information Sharing Among Doctors at Different Location

WWW is commonly known as the Web and it is a system of interlinked hypertext documents accessed via the Internet. With a web browser, web pages containing text, images, video and other multimedia can be viewed. It also provides platform for navigation between and via the hyperlinks. The WWW component of the architecture focuses on eliminating geographical barriers in the diagnosis of heart failure patients. It also enables the deployment of a centralized database which enhances the management of patients' medical records and provides a means for communication on patients' information among medical experts. The conceptual diagram of the proposed website for communication on diagnosis of heart failure disease is presented in Figure 5. The home page provides links via which other modules that constitute the entire system could be accessed. The site consists of modules for registration and authentication, record entry and update, diagnostics and report. 


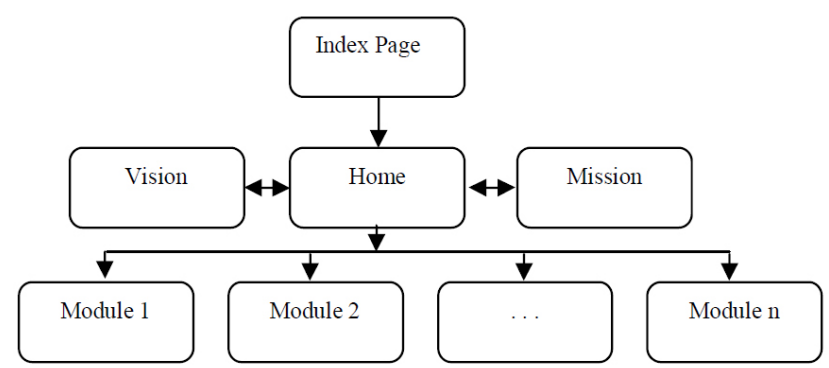

Figure 5: A Conceptual view of the website for the proposed system

\section{System implementation, experimental study and findings}

The proposed system was implemented in a web environment characterized by windows XP Operating System (OS) on a Pentium 4 processor running at $1.6 \mathrm{GHZ}$ processor speed on 512 MB Random Access Memory (RAM), and 100 GB Hard Disk Drive (HDD). The system consists of three independently installed and configured components; namely front tier, server application and the knowledge base server (back-end tier). The front-end is coded with HTML, AJAX, and JavaScript programming languages. The HTML tags were employed to structure the outlook of the web pages in the system, while Java Script code were introduced to authenticate and validate the data entered on the designed web pages. AJAX codes were employed to download data from the server. Both HTML, AJAX, and JavaScript) provide a wide range of capabilities with several free editors. The middle tier is an intermediary between the front and the back ends and provides medium for presenting user's request to the server. It requires a network connection for the knowledge base server at the back end. The middle-tier was implemented using Hypertext Preprocessor (PHP) Scripting language which runs about 20 times faster than Java and provide speedy and extremely easy to adopt environment for development of very complex web applications. The back end was implemented using MySQL Database Management System (DBMS) and hosts the experts' knowledge. The choice of MySQL is based on its fast relational database management capability and functionalities.

The proposed system is designed to enable prospective user to register and create an account by supplying valid details as specified on the account set up page presented in Figure 6. The Login page enables registered users to access the system by providing correct login details (username and password) with pre-assigned privileges. Upon successful login, the Home Page that holds the information on the risks factors and indicators of Heart Failure disease is displayed. The Home page also provides links to pages for patient's profile entry and update and diagnosis. It also leads to the history page on which a brief summary of previous diagno- sis results is presented at a glance.

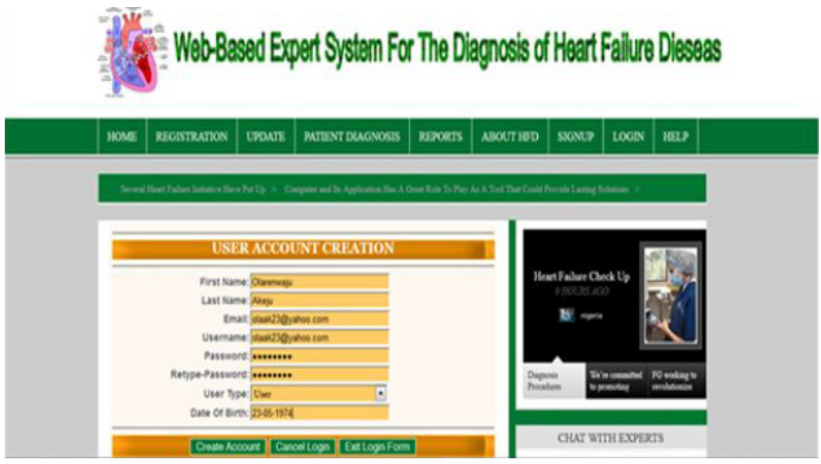

Figure 6: User Account Creation Page

Table 4 shows the figures which represent the ratings of forty (40) randomly selected heart failure patients at the State Specialist Hospital, Akure, Nigeria. The figures were collected via extensive interaction (interview) with some patients and medical experts and examination of related records in the Hospital. Patients' levels of Heart Failure Disease were evaluated by domain experts based on signs, symptoms and results of laboratory investigations. The intensity of the signs, symptoms and investigation was rated as healthy (0), mild (1), moderate (2), severe (3) and very severe (4).

Table 5 presents the obtained triangular fuzzy numbers (TFN) of the figures presented in Table 4. A TFN value represents the mild, moderate, severe or very severe level for a diagnosis by using the fuzzifier based on Equation (2) for a variable. For example, during diagnosis, a cardiologist may assign a value of 3 out of 4 to Dypsnea While Climbing (DC) attribute to a patient who complained of tiredness while climbing the stair case. The degree of severity which represents the triangular fuzzy number is then evaluated as 0.5 . The rule base evaluation for the patient with ID " 001 " is presented in Table 5 .

It is revealed in Table 6 that seven rules were fired for patient ID 001. The seven rules generated non-zero minimum values from the system and for each of the linguistic variables: mild, moderate, severe, and very severe, their respective output membership function strength falls within the range (0 1) while the result of the diagnosis is computed using RSS inference technique as follows:
Mild $=\sqrt{R 7^{2}}=\sqrt{0.25^{2}}=0.25$
Moderate $=\sqrt{R 2^{2}}=\sqrt{0.25^{2}}=0.25$
Severe $=\sqrt{R 6^{2}+R 15^{2}+R 20^{2}}$
$=\sqrt{0.50^{2}+0.25^{2}+0.50^{2}}=0.75$
Very Severe $=\sqrt{R 10^{2}+R 17^{2}}$
$=\sqrt{0.50^{2}+0.25^{2}}=0.56$ 
Table 4: Rating of Patients with Respect to Heart Failure Diagnosis Attributes

\begin{tabular}{|c|c|c|c|c|c|c|c|c|c|c|c|c|c|c|c|}
\hline Pat. ID & RD & OP & ND & DE & DC & HR & VE & LC & WZ & HS & AP & IP & BP & CR & $\mathbf{Z F}$ \\
\hline 001 & 4 & 3 & 3 & 2 & 4 & 3 & 2 & 1 & 1 & 2 & 2 & 4 & 2 & 1 & 2 \\
\hline 002 & 3 & 4 & 4 & 2 & 1 & 0 & 3 & 2 & 3 & 0 & 2 & 2 & 1 & 1 & 0 \\
\hline 003 & 1 & 2 & 1 & 2 & 1 & 2 & 3 & 2 & 0 & 2 & 1 & 2 & 1 & 3 & 3 \\
\hline 004 & 4 & 1 & 3 & 2 & 1 & 3 & 3 & 2 & 3 & 0 & 2 & 2 & 2 & 1 & 3 \\
\hline 005 & 0 & 3 & 2 & 1 & 4 & 2 & 0 & 0 & 0 & 3 & 2 & 3 & 4 & 2 & 2 \\
\hline 006 & 2 & 3 & 3 & 2 & 1 & 4 & 2 & 2 & 3 & 3 & 2 & 2 & 2 & 2 & 1 \\
\hline 007 & 1 & 4 & 3 & 2 & 1 & 2 & 2 & 2 & 3 & 1 & 0 & 2 & 1 & 2 & 2 \\
\hline 008 & 3 & 4 & 2 & 2 & 2 & 1 & 2 & 2 & 1 & 3 & 0 & 0 & 1 & 0 & 2 \\
\hline 009 & 3 & 3 & 2 & 3 & 1 & 2 & 3 & 3 & 2 & 1 & 0 & 2 & 2 & 2 & 1 \\
\hline 010 & 2 & 2 & 1 & 2 & 1 & 3 & 1 & 3 & 1 & 0 & 1 & 2 & 1 & 3 & 3 \\
\hline 011 & 4 & 2 & 2 & 2 & 1 & 3 & 2 & 2 & 3 & 1 & 0 & 2 & 2 & 1 & 0 \\
\hline 012 & 4 & 1 & 2 & 2 & 2 & 1 & 2 & 1 & 1 & 2 & 2 & 3 & 2 & 2 & 3 \\
\hline 013 & 3 & 4 & 3 & 1 & 1 & 0 & 3 & 2 & 3 & 0 & 2 & 4 & 2 & 0 & 0 \\
\hline 014 & 3 & 1 & 1 & 2 & 0 & 4 & 4 & 3 & 0 & 2 & 1 & 2 & 1 & 1 & 0 \\
\hline 015 & 2 & 1 & 3 & 2 & 1 & 3 & 3 & 2 & 3 & 0 & 2 & 2 & 2 & 1 & 1 \\
\hline 016 & 0 & 3 & 1 & 1 & 2 & 2 & 2 & 1 & 0 & 1 & 0 & 3 & 4 & 2 & 2 \\
\hline 017 & 3 & 2 & 3 & 1 & 1 & 2 & 4 & 2 & 3 & 0 & 2 & 2 & 3 & 2 & 1 \\
\hline 018 & 1 & 2 & 1 & 2 & 1 & 0 & 3 & 2 & 3 & 0 & 2 & 2 & 1 & 1 & 0 \\
\hline 019 & 0 & 2 & 2 & 1 & 2 & 3 & 1 & 2 & 0 & 0 & 0 & 1 & 4 & 1 & 2 \\
\hline 020 & 4 & 2 & 4 & 2 & 2 & 0 & 0 & 1 & 3 & 0 & 1 & 2 & 3 & 2 & 4 \\
\hline 021 & 2 & 4 & 1 & 2 & 4 & 2 & 3 & 1 & 0 & 0 & 1 & 3 & 2 & 1 & 1 \\
\hline 022 & 1 & 3 & 2 & 3 & 0 & 0 & 2 & 3 & 3 & 0 & 2 & 3 & 2 & 2 & 3 \\
\hline 023 & 3 & 4 & 4 & 2 & 2 & 1 & 4 & 2 & 1 & 0 & 0 & 0 & 2 & 1 & 2 \\
\hline 024 & 3 & 2 & 1 & 3 & 1 & 2 & 4 & 0 & 0 & 2 & 3 & 4 & 2 & 4 & 1 \\
\hline 025 & 2 & 3 & 1 & 1 & 3 & 0 & 2 & 1 & 0 & 3 & 1 & 2 & 1 & 2 & 3 \\
\hline 026 & 1 & 1 & 4 & 2 & 1 & 3 & 1 & 2 & 3 & 4 & 3 & 1 & 0 & 0 & 2 \\
\hline 027 & 2 & 4 & 1 & 3 & 4 & 2 & 2 & 0 & 3 & 0 & 0 & 0 & 1 & 1 & 4 \\
\hline 028 & 3 & 2 & 0 & 2 & 0 & 1 & 2 & 3 & 1 & 3 & 2 & 3 & 1 & 1 & 2 \\
\hline 029 & 0 & 4 & 2 & 1 & 2 & 1 & 0 & 1 & 2 & 1 & 0 & 2 & 4 & 1 & 1 \\
\hline 030 & 3 & 2 & 3 & 4 & 3 & 4 & 2 & 3 & 4 & 1 & 0 & 3 & 3 & 4 & 1 \\
\hline 031 & 4 & 2 & 2 & 2 & 1 & 3 & 2 & 2 & 3 & 1 & 0 & 2 & 2 & 1 & 0 \\
\hline 032 & 3 & 1 & 2 & 3 & 1 & 1 & 2 & 1 & 0 & 2 & 0 & 3 & 2 & 0 & 3 \\
\hline 033 & 4 & 4 & 1 & 2 & 1 & 0 & 3 & 1 & 2 & 1 & 0 & 4 & 2 & 4 & 3 \\
\hline 034 & 4 & 2 & 1 & 2 & 0 & 4 & 4 & 3 & 0 & 2 & 1 & 2 & 1 & 2 & 2 \\
\hline 035 & 3 & 2 & 3 & 4 & 0 & 3 & 0 & 0 & 3 & 0 & 2 & 1 & 4 & 1 & 1 \\
\hline 036 & 0 & 3 & 1 & 1 & 2 & 2 & 2 & 1 & 0 & 1 & 0 & 3 & 4 & 2 & 2 \\
\hline 037 & 3 & 2 & 3 & 1 & 1 & 2 & 4 & 2 & 3 & 0 & 2 & 2 & 3 & 2 & 1 \\
\hline 038 & 1 & 2 & 1 & 2 & 1 & 0 & 3 & 2 & 3 & 0 & 2 & 2 & 1 & 1 & 0 \\
\hline 039 & 2 & 4 & 2 & 3 & 2 & 3 & 3 & 2 & 1 & 1 & 0 & 1 & 3 & 4 & 1 \\
\hline 040 & 3 & 3 & 1 & 0 & 2 & 0 & 0 & 1 & 3 & 0 & 1 & 0 & 3 & 2 & 2 \\
\hline
\end{tabular}

The fuzzy set from the RSS is then defuzzified to obtain the crisp output of the inference engine using the discrete Centroid of Area $(\mathrm{CoA})$ technique as follows: Crisp Output $=\frac{(0.25 * 0.3)+(0.25 * 0.5)+(0.75 * 0.7)+(0.56 * 0.9)}{(0.25)+(0.25)+(0.75)+(0.56)}$
$=0.69$

Based on Table 4, this output indicates that the patient 'D001' has possibility of severe heart failure. The judgments for the authenticity of these results were based on cognitive and emotional considerations. Cognitive consideration involves the use of patients' alcohol, smoking, family and other related history while emotional consideration involves the use of such factors as job, social and family induced stresses which may necessitate bed rest. The computed crisp outputs for all the fired rules for the other thirty nine patients fall within the range predefined by medical experts. Figure 7 shows the percentage of the diagnosis status based on obtained crisp values for the 40 patients. 
Table 5: Triangular Fuzzy Numbers of the Data in Table 4

\begin{tabular}{|c|c|c|c|c|c|c|c|c|c|c|c|c|c|c|c|}
\hline PAT & RD & OP & ND & DE & DC & HR & VE & LC & WZ & HS & AP & IP & BP & CR & $\mathbf{Z F}$ \\
\hline 001 & 0.75 & 0.50 & 0.50 & 0.25 & 0.75 & 0.50 & 0.25 & 0.00 & 0.00 & 0.00 & 0.25 & 0.75 & 0.25 & 0.00 & 0.25 \\
\hline 002 & 0.50 & 0.75 & 0.75 & 0.25 & 0.00 & 0.00 & 0.50 & 0.25 & 0.50 & 0.00 & 0.25 & 0.25 & 0.00 & 0.00 & 0.00 \\
\hline 003 & 0.00 & 0.25 & 0.00 & 0.25 & 0.00 & 0.25 & 0.50 & 0.25 & 0.00 & 0.25 & 0.00 & 0.25 & 0.00 & 0.50 & 0.50 \\
\hline 004 & 0.75 & 0.00 & 0.50 & 0.25 & 0.00 & 0.50 & 0.50 & 0.25 & 0.50 & 0.00 & 0.25 & 0.25 & 0.25 & 0.00 & 0.50 \\
\hline 005 & 0.00 & 0.50 & 0.25 & 0.00 & 0.75 & 0.25 & 0.00 & 0.00 & 0.00 & 0.50 & 0.25 & 0.50 & 0.75 & 0.25 & 0.25 \\
\hline 006 & 0.25 & 0.50 & 0.50 & 0.25 & 0.00 & 0.75 & 0.25 & 0.25 & 0.50 & 0.50 & 0.25 & 0.25 & 0.25 & 0.25 & 0.00 \\
\hline 007 & 0.00 & 0.75 & 0.50 & 0.25 & 0.00 & 0.25 & 0.25 & 0.25 & 0.50 & 0.00 & 0.00 & 0.25 & 0.00 & 0.25 & 0.25 \\
\hline 008 & 0.50 & 0.75 & 0.25 & 0.25 & 0.25 & 0.00 & 0.25 & 0.25 & 0.00 & 0.50 & 0.00 & 0.00 & 0.00 & 0.00 & 0.25 \\
\hline 009 & 0.50 & 0.50 & 0.25 & 0.50 & 0.00 & 0.25 & 0.50 & 0.50 & 0.25 & 0.00 & 0.00 & 0.25 & 0.25 & 0.25 & 0.00 \\
\hline 010 & 0.25 & 0.25 & 0.00 & 0.25 & 0.00 & 0.50 & 0.00 & 0.50 & 0.00 & 0.00 & 0.00 & 0.25 & 0.00 & 0.50 & 0.50 \\
\hline 011 & 0.75 & 0.25 & 0.25 & 0.25 & 0.00 & 0.50 & 0.25 & 0.25 & 0.50 & 0.00 & 0.00 & 0.25 & 0.25 & 0.00 & 0.00 \\
\hline 012 & 0.75 & 0.00 & 0.25 & 0.25 & 0.25 & 0.00 & 0.25 & 0.00 & 0.00 & 0.25 & 0.25 & 0.50 & 0.25 & 0.25 & 0.50 \\
\hline 013 & 0.50 & 0.75 & 0.50 & 0.00 & 0.00 & 0.00 & 0.50 & 0.25 & 0.50 & 0.00 & 0.25 & 0.75 & 0.25 & 0.00 & 0.00 \\
\hline 014 & 0.50 & 0.00 & 0.00 & 0.25 & 0.00 & 0.75 & 0.75 & 0.50 & 0.00 & 0.25 & 0.00 & 0.25 & 0.00 & 0.00 & 0.00 \\
\hline 015 & 0.25 & 0.00 & 0.50 & 0.25 & 0.00 & 0.50 & 0.50 & 0.25 & 0.50 & 0.00 & 0.25 & 0.25 & 0.25 & 0.00 & 0.00 \\
\hline 016 & 0.00 & 0.50 & 0.00 & 0.00 & 0.25 & 0.25 & 0.25 & 0.00 & 0.00 & 0.00 & 0.00 & 0.50 & 0.75 & 0.25 & 0.25 \\
\hline 017 & 0.50 & 0.25 & 0.50 & 0.00 & 0.00 & 0.25 & 0.75 & 0.25 & 0.75 & 0.00 & 0.25 & 0.25 & 0.50 & 0.25 & 0.00 \\
\hline 018 & 0.00 & 0.25 & 0.00 & 0.25 & 0.00 & 0.00 & 0.50 & 0.25 & 0.50 & 0.00 & 0.25 & 0.25 & 0.00 & 0.00 & 0.00 \\
\hline 019 & 0.00 & 0.25 & 0.25 & 0.00 & 0.25 & 0.50 & 0.00 & 0.25 & 0.00 & 0.00 & 0.00 & 0.00 & 0.75 & 0.00 & 0.25 \\
\hline 020 & 0.75 & 0.25 & 0.75 & 0.25 & 0.25 & 0.00 & 0.00 & 0.00 & 0.50 & 0.00 & 0.00 & 0.25 & 0.50 & 0.25 & 0.75 \\
\hline 021 & 0.25 & 0.75 & 0.00 & 0.25 & 0.75 & 0.25 & 0.50 & 0.00 & 0.00 & 0.00 & 0.00 & 0.50 & 0.25 & 0.00 & 0.00 \\
\hline 022 & 0.00 & 0.50 & 0.25 & 0.50 & 0.00 & 0.00 & 0.25 & 0.50 & 0.50 & 0.00 & 0.25 & 0.50 & 0.25 & 0.25 & 0.50 \\
\hline 023 & 0.50 & 0.75 & 0.75 & 0.25 & 0.25 & 0.00 & 0.75 & 0.25 & 0.00 & 0.00 & 0.00 & 0.00 & 0.25 & 0.00 & 0.25 \\
\hline 024 & 0.50 & 0.25 & 0.00 & 0.50 & 0.00 & 0.25 & 0.75 & 0.00 & 0.00 & 0.25 & 0.50 & 0.75 & 0.25 & 0.75 & 0.00 \\
\hline 025 & 0.25 & 0.50 & 0.00 & 0.00 & 0.50 & 0.00 & 0.25 & 0.00 & 0.00 & 0.50 & 0.00 & 0.25 & 0.00 & 0.25 & 0.50 \\
\hline 026 & 0.00 & 0.00 & 0.75 & 0.25 & 0.00 & 0.50 & 0.00 & 0.25 & 0.50 & 0.75 & 0.50 & 0.00 & 0.00 & 0.00 & 0.25 \\
\hline 027 & 0.25 & 0.75 & 0.00 & 0.50 & 0.75 & 0.25 & 0.25 & 0.00 & 0.50 & 0.00 & 0.00 & 0.00 & 0.00 & 0.00 & 0.75 \\
\hline 028 & 0.50 & 0.25 & 0.00 & 0.25 & 0.00 & 0.00 & 0.25 & 0.50 & 0.00 & 0.50 & 0.25 & 0.50 & 0.00 & 0.00 & 0.25 \\
\hline 029 & 0.00 & 0.75 & 0.25 & 0.00 & 0.25 & 0.00 & 0.00 & 0.00 & 0.25 & 0.00 & 0.00 & 0.25 & 0.45 & 0.00 & 0.00 \\
\hline 030 & 0.50 & 0.25 & 0.50 & 0.75 & 0.50 & 0.75 & 0.25 & 0.50 & 0.75 & 0.00 & 0.00 & 050 & 0.50 & 0.75 & 0.00 \\
\hline 031 & 0.75 & 0.25 & 0.25 & 0.25 & 0.00 & 0.50 & 0.25 & 0.25 & 0.50 & 0.00 & 0.00 & 0.25 & 0.25 & 0.00 & 0.00 \\
\hline 032 & 0.50 & 0.00 & 0.25 & 0.50 & 0.00 & 0.00 & 0.25 & 0.00 & 0.00 & 0.25 & 0.00 & 0.50 & 0.25 & 0.00 & 0.50 \\
\hline 033 & 0.75 & 0.75 & 0.00 & 0.25 & 0.00 & 0.00 & 0.50 & 0.00 & 0.25 & 0.00 & 0.00 & 0.75 & 0.25 & 0.75 & 0.50 \\
\hline 034 & 0.75 & 0.25 & 0.00 & 0.25 & 0.00 & 0.75 & 0.75 & 0.50 & 0.00 & 0.25 & 0.00 & 0.25 & 0,00 & 0.25 & 0.25 \\
\hline 035 & 0.50 & 0.25 & 0.50 & 0.75 & 0.00 & 0.50 & 0.00 & 0.00 & 0.50 & 0.00 & 0.25 & 0.00 & 0.75 & 0.00 & 0.00 \\
\hline 036 & 0.00 & 0.50 & 0.00 & 0.00 & 0.25 & 0.25 & 0.25 & 0.00 & 0.00 & 0.00 & 0.00 & 0.25 & 0.75 & 0.25 & 0.25 \\
\hline 037 & 0.50 & 0.25 & 0.50 & 0.00 & 0.00 & 0.25 & 0.75 & 0.25 & 0.50 & 0.00 & 0.25 & 0.25 & 0.50 & 0.25 & 0.00 \\
\hline 038 & 0.00 & 0.25 & 0.00 & 0.25 & 0.00 & 0.00 & 0.50 & 0.25 & 0.50 & 0.00 & 0.25 & 0.25 & 0.00 & 0.00 & 0.00 \\
\hline 039 & 0.25 & 0.75 & 0.25 & 0.50 & 0.25 & 0.50 & 0.50 & 0.25 & 0.00 & 0.00 & 0.00 & 0.00 & 0.50 & 0.75 & 0.00 \\
\hline 040 & 0.50 & 0.50 & 0.00 & 0.00 & 0.25 & 0.00 & 0.00 & 0.00 & 0.50 & 0.00 & 0.00 & 0.00 & 0.50 & 0.25 & 0.25 \\
\hline
\end{tabular}

A formative evaluation of the system based on the view and responses of 50 out of 63 randomly selected experts at Specialist Hospital, Akure, Nigeria on diagnosis of heart failure disease was conducted in line with some indices. The view and responses were sampled using questionnaire and interview methods. The selections of the respondents were freely done without any form of bias. The first section of the questionnaire focused on the relevant information about the respondent which includes name and year of experience. The second section featured the evaluation indices such as Published by Sciedu Press accessibility, availability, level of interaction, menu driven support, support for diagnosis and decision support. The indices were rated on a scale of 5, 4, 3, 2 and 1 for "Excellent", "Very Good", "Good", "Average" and "Poor" respectively. Summaries of the Average ratings of the system by the 50 experts are presented 13 in Table 7 (A=Accessibility, $\mathrm{B}=$ Availability, $\mathrm{C}=$ Level of Interaction, $\mathrm{D}=$ Menu Driven support, $\mathrm{E}=$ Support for Diagnosis, $\mathrm{F}=$ Usefulness for Decision Making). 
Table 6: Rule Base Evaluation for Patient with ID 001

\begin{tabular}{|c|c|c|c|c|c|c|c|c|c|c|c|c|c|c|c|c|c|}
\hline Rule & RD & OP & ND & DE & DC & HR & VE & LC & WZ & HS & AP & IP & BP & CR & $\mathbf{Z F}$ & Conclusion & $\begin{array}{l}\text { Non-Zero } \\
\text { Min. Value }\end{array}$ \\
\hline 2 & - & - & - & - & - & 0.25 & 0.25 & - & - & 0.25 & - & - & - & - & - & Moderate & 0.25 \\
\hline 6 & 0.50 & - & 0.75 & - & - & - & 0.50 & - & - & - & & - & - & - & - & Severe & 0.50 \\
\hline 7 & 0.25 & 0.25 & - & - & - & - & 0.50 & - & - & - & - & 0.25 & - & - & 0.50 & Mild & 0.25 \\
\hline 10 & - & - & - & - & - & 0.50 & 0.75 & - & 0.50 & - & - & 0.50 & 0.50 & - & 0.50 & V. Severe & 0.50 \\
\hline 15 & - & - & - & 0.75 & 0.50 & - & - & - & - & - & 0.25 & - & 0.75 & 0.25 & 0.25 & Severe & 0.25 \\
\hline 17 & - & - & 0.25 & - & - & - & 0.25 & - & 0.50 & - & - & - & - & - & 0.75 & V. Severe & 0.25 \\
\hline 20 & 0.75 & - & 0.75 & - & - & - & 0.50 & - & 0.75 & - & - & - & - & - & - & Severe & 0.50 \\
\hline
\end{tabular}

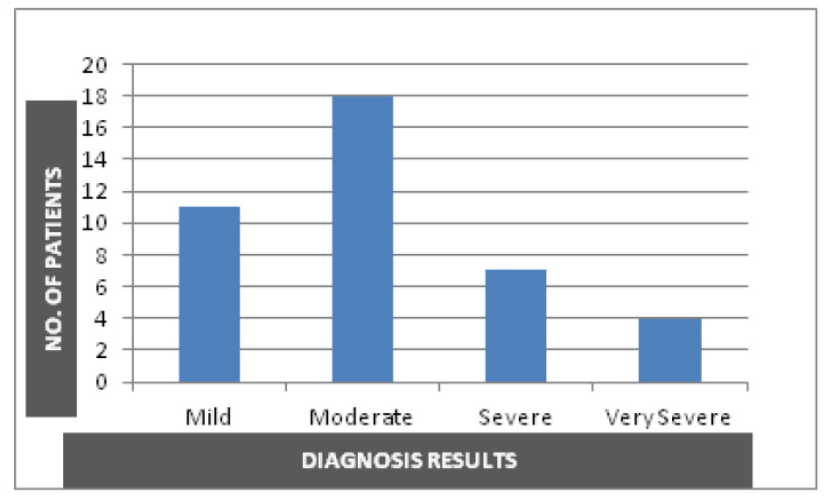

Figure 7: Diagnosis Status for the 40 Patients

Table 7: Experts Evaluation Results

\begin{tabular}{ll}
\hline Index & Average Rating \\
\hline A & 2.17 \\
B & 3.59 \\
C & 4.01 \\
D & 3.71 \\
E & 4.23 \\
F & 4.05 \\
\hline
\end{tabular}

According to Tables 7, all the 50 surveyed experts gave 2.17 (Average), 3.59 (Good), 4.01 (Very Good), 3.71 (Good), 4.23 (Very Good) and 4.05 (Very Good) average ratings for the proposed system in term of the "accessibility", "availability", "level of interaction", "menu driven support", "support for diagnosis" and "usefulness for decision making" respectively. These results revealed manageable differences in the ratings of the indices and also denote encouraging and acceptable performances to the experts. The "Average" rat- ing on "Accessibility" is attributed to the weak nature of the system's Internet platform.

\section{Conclusions}

This research work has explored and applied fuzzy logic concept to medical diagnosis of heart failure disease due to its strength in providing accurate solutions when several decision variables are involved. The system runs on the Internet for global access to patients' record and collaboration among experts. The design and implementation of the system involves a three tier architecture comprising of the Front-end, Middle-end and Back-end. The front-end engine is the Internet platform while the middle-end contains the Application Engine characterized by insertion, update and query transactions. The back-end engine on its own houses the system's database. With the proposed system, it is possible to maintain easy access and maintenance of patients and experts profiles with great emphasis on system personalization. The system provides adequate security and privacy mechanisms with a view to guaranteeing the integrity of its database. The profile of the administrator and users of the system are adequately authenticated at the application's levels of entry. The satisfactory performance of the system based on ratings by experts has been reported.

Future research aims at improving the performance ratings especially in areas of "accessibility" through enhanced and fortified modules and improvement of the Front end (Internet) Engine. Consideration will also be given to alternative platform, front-end, middleend and back-end engines with more emphasis on open sourced applications that are versatile and robust with improved usage, privacy and security features.

\section{References}

[1] The Patient Education Institute (X-Plain.com), Inc. (c) 1995-2010, http://www.XPlain.com. This Page was last reviewed: 06/21/2010. Available from: http://www . webmd.com/

[2] Kumosani T.A., Mohamed N.A. and Archana I. Cardiovascular diseases in Saudi Arabia. Prime Research on Medicine (PROM). 2011;
1: $1-6$.

[3] Ali A. and Mehli, N. A Fuzzy Expert System for Heart Disease Diagnosis. Proceedings of the International Multi-Conference of Engineers and Computer Scientists. 2010; 1. IMECS, March 17-19, Hong Kong.

[4] Saba T., Saleh A. and Amjad R. Expert System for Offline Clinical Guidelines and Treatment Life Science Journal. 2012; 9(4).

[5] Gath S. J., Kulkarni R. V. A Review: Expert System for Diagnosis 
of Myocardial Infarction. International Journal of Computer Science and Information Technologies. 2012; 3(6): 5315-5321.

[6] Salam A.M., Mohamed R. and Rania A.H. A Case Based Expert System for Supporting Diagnosis of Heart Diseases. AIML Journal. 2005; 5(1).

[7] Celli B.R. and MacNee W. Standards for Diagnosis and Treatment of Patients with COPD: a Summary of ATS/ERS Position Paper. European Respiratory Journal. 2004; 23: 932-946. http: //dx.doi.org/10.1183/09031936.04.00014304

[8] European Society of Cardiology (ESC). Guidelines for Diagnosis and Treatment of Acute and Chronic Heart Failure. European heart journal. 2012; 33: 1787-1847. PMid:22611136

[9] Amin S.U, Umar K.A and Rizwan B. 2013. Data Mining in Clinical Decision Support Systems for Diagnosis, Prediction and Treatment of Heart Disease. International Journal of Advanced Research in Computer Engineering \& Technology (IJARCET), Volume 2, Issue 1, WebMD (2012). Available from: http://www.webmd.co $\mathrm{m} /$ content/article/51/40699.htm. Date Retrieved: May 20, 2012.

[10] Gayathri. P and Jaisankar N. Comprehensive Study of Heart Disease Diagnosis Using Data Mining and Soft Computing Techniques. International Journal of Engineering and Technology (IJET). 2013; 5(3).

[11] Hannan S.A., Manza R.R. and Ramteke R.J. Generalized Regression Neural Network and Radial Basis Function for Heart Disease Diagnosis, International Journal of Computer Applications. 2010; $7(13)$.

[12] Akinyokun O.C. and Adeniyi O.A. Experimental Study of Intelligent Computer Aided Diagnostics and Therapy. AMSE Journal of Modeling, Simulation and Control. 1991; 27(3): 1-20.

[13] Olabiyisi S.O. and Omidiora E.O. A Decision Support System Model for Diagnosing Tropical Diseases Using Fuzzy Logic. African Journal of Computing \& ICT. 2011; 4.
[14] Yan H., Yingtao J., Jun Z., Chenglin P. and Qinghui L. A Multilayer Perceptron-Based Medical Decision Support System for Heart Disease Diagnosis. Expert Systems with Applications. 2006; 30: 272281. http://dx.doi.org/10.1016/j.eswa.2005.07.022

[15] Hudson D.L. and Cohen M.E. Use of Intelligent Agents in the Diagnosis of Cardiac Disorders. Computers in Cardiology. 2002; 29: 633-636.

[16] Adeli A. and Mehdi N. A Fuzzy Expert System for Heart Disease Diagnosis. Proceedings of International Multi-Conference of Engineers and Computer Scientists (IMECS2010), Hong Kong. 2010.

[17] Obot, O.U. 2007. A Neuro-Fuzzy Expert System Model for the Diagnosis and Therapy of Cardiovascular Diseases (A Case Study of Heart Failure). PhD Thesis, Federal University of Technology, Akure, Nigeria.

[18] Akinyokun O.C., Obot O.U., Uzoka F.E. and Andy J.J. A NeuroFuzzy Decision Support System for Diagnosis of Heart Failure. Journal of Medical and Care Compunetics, ISO Press. 2010; 6(10): 231-244.

[19] Taboada M., Des J., Mira J. and Marín R. 1992. Diagnosis Systems in Medicine with Reusable Knowledge Components. IEEE Transaction on Intelligent Systems in Biology, November/December 2001.

[20] Abraham B. and Nath S. Hybrid Intelligent Systems Design For Health Care Delivery-A Review of a Decade Research. Journal of the School of Computing and Information Technology, Monash, Australia. 2003; 1: 1-37.

[21] Chakkrit S. and Michael B. Personal Health Assistance Service Expert System. World Academic of Science, Engineering and Technology. 2007; 32: 186 -192.

[22] Moein S., Monadjemi S.A. and Moallem P. A Novel Fuzzy - Neural Based Medical Diagnosis System. World Academy of Science, Eng. and Tech. 2008; 37: 157-161.

[23] Djam X.Y., Wajiga G.M., Kimbi, Y.H. and Blama, N.V. A Fuzzy Expert System for the Management of Malaria. International Journal of Pure and Applied Science and Technology. 2011; 5(2): 84-108. 